> Les kinines sont des peptides autacoïdes (hormones locales) et des neuromédiateurs centraux impliqués dans le contrôle cardiovasculaire, I'inflammation et la douleur. Leurs effets sont relayés par deux types de récepteurs couplés aux protéines $G$ : un récepteur $B_{2}$, constitutif, et un récepteur $B_{1}$, inductible en présence de cytokines, d'endotoxines ou de lésions tissulaires. Alors que le récepteur $B_{2}$ contribue aux effets bénéfiques des inhibiteurs de l'enzyme de conversion de l'angiotensine-I utilisés dans le traitement des maladies cardiovasculaires, il participe à la phase aiguë de l'inflammation et de la douleur somatique et viscérale. Le récepteur $B_{1}$ participe, quant à lui, à la phase chronique de ces réponses, et jouerait un rôle stratégique dans les maladies ayant une forte composante immune telles que l'arthrite rhumatoïde, la sclérose en plaques, le choc septique ou le diabète. II posséderait également une dualité fonctionnelle, se manifestant par une action protectrice (vis-à-vis de la sclérose en plaques et du choc septique, par exemple) ou délétère (dans le cas de la douleur et de l'inflammation). Ainsi, l'utilisation d'antagonistes de ces récepteurs comme agents thérapeutiques nécessite une évaluation rigoureuse de leurs effets. <

\section{Dualité \\ fonctionnelle \\ des récepteurs \\ des kinines en \\ physiopathologie}

Bichoy H. Gabra, Réjean Couture, Pierre Sirois

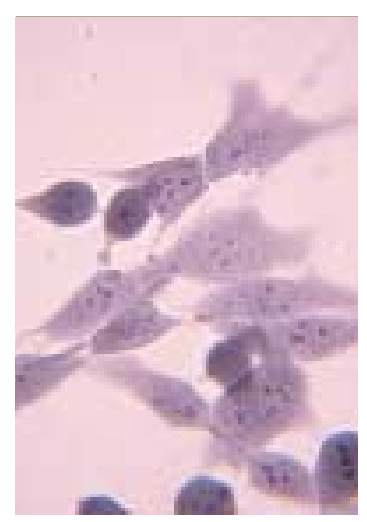

Le système kallicréinekinine (SKK) est un système peptidergique complexe comprenant les enzymes de synthèse, appelées kallicréines, leurs substrats, les kininogènes et les peptides vasoactifs, appelés kinines. Ces derniers sont des médiateurs importants impliqués dans une variété d'effets biologiques incluant l'homéostasie cardiovasculaire, l'inflammation et la nociception [1, 2]. En effet, ces peptides autacoïdes sont parmi les premiers médiateurs libérés dans les tissus lésés à partir des kininogènes sous l'action de la kallicréine plasmatique (activée tôt dans la cascade de coagulation) ou de la kallicréine tissulaire (activée par des protéases produites au sein de la lésion) [3]. Les kinines peuvent également être produites à la suite de stimulus nocifs, et des données expérimentales suggèrent qu'elles sont emmagasinées dans des neurones du système nerveux central au niveau duquel elles pourraient jouer le rôle de neuromédiateurs dans certaines fonctions cérébrales, en particulier dans le contrôle des informations nociceptives et de la pression artérielle [4].

Tableau I. Structure primaire des kinines chez les mammifères. 
$(\rightarrow) \mathrm{m} / \mathrm{s}$

$1996, n^{\circ} 5$,

p. 582
Métabolisme des kinines

\section{Formation des kinines}

Les kinines appartiennent à une petite famille de peptides possédant 9 à 11 acides aminés, incluant la bradykinine (BK), la kallidine (KD; Lys-BK), la T-kinine (Ile-Ser-BK) et leurs métabolites actifs dépourvus de l'arginine en position carboxy-terminale $(\rightarrow)$ (Tableau I). La BK et la KD sont les produits de deux voies biochimiques, l'une sanguine et l'autre tissulaire (Figure IA). La formation de BK est amorcée par l'activation du facteur de Hageman (facteur XII de la coagulation) lorsque le sang entre en contact avec des surfaces possédant des charges négatives telles que les composantes de la matrice cellulaire (collagène, protéoglycanes et héparine) ou d'autres particules chargées négativement (urate, phospholipides acides, sulfate de cholestérol, sulfate de chondroïtine, carragénine et lipopolysaccharides). La pré-kallicréine plasmatique, associée au kininogène de haut poids moléculaire (KHPM, 110 kDa), est convertie par le facteur de Hageman activé en kallicréine. Le traitement du KHPM par la kallicréine ainsi active libère la BK (Figure IA). Dans les tissus, des enzymes protéolytiques activent la kallicréine tissulaire qui agit sur le kininogène de bas poids moléculaire (KBPM, $70 \mathrm{kDa}$ ) pour former la KD, sauf chez le rat où la BK et non la KD est produite [3].

Un seul gène code pour la kallicréine plasmatique, tandis que la kallicréine tissulaire est codée par une famille multigénique [3]. La T-kinine a été identifiée exclusivement chez le rat où elle est produite par une enzyme de synthèse encore inconnue. Le T-kininogène se présente sous deux isoformes de protéines, T-I et T-II kininogènes, possédant une homologie de $96 \%$ et codées par deux gènes différents. Les KHPM et KBPM proviennent d'un seul gène, le gène $K$, et résultent de la transcription de deux ARNm différents. Les gènes $K$ et $T$ des kininogènes proviennent d'une duplication d'un gène ancestral, suivie dans le cas du rat par une duplication additionnelle aboutissant à la formation des deux gènes des T-kininogènes [4] (Figure IB).

\section{Dégradation des kinines}

Les kinines subissent une dégradation métabolique rapide par des amino-, carboxy- et endopeptidases appelées kininases, trouvées dans le sang, les tissus et les liquides biologiques, qui aboutit à la production de plusieurs métabolites actifs et inactifs (Figure 2A). La demi-vie de la BK est inférieure à 30 secondes dans le plasma [5]. Les enzymes les plus importantes dans le métabolisme des kinines sont les kininases I et II. La kininase I est représentée par la carboxypeptidase $\mathrm{N}$ du plasma (CPN) et la carboxypeptidase $M$ de la membrane cellulaire (CPM), qui clivent l'arginine en position carboxy-terminale pour produire la desArg ${ }^{9}$-BK (DBK), la desArg ${ }^{10}$-KD ou la desArg ${ }^{11}-T-$ kinine. Ces enzymes sont particulièrement importantes, car elles produisent les seuls métabolites de la BK, de la KD ou de la T-kinine ayant une activité biologique significative. II existe également deux types de kininase II, l'enzyme de conversion de l'angiotensine-I ( $(C A)$ et l'endopeptidase neutre 24.11 ( $E P N$, également appelée enképhalinase). Les deux enzymes clivent le dipeptide $\mathrm{Phe}^{8}-\mathrm{Arg}^{9}$ en position carboxy-terminale de la BK pour produire le métabolite inactif BK-(1-7). L'ECA enlève également le dipeptide $\mathrm{Ser}^{6}$ $\mathrm{Pro}^{7}$ de la BK-(1-7) pour donner la BK-(1-5), qui est le

\section{A FORMATION DES KININES}
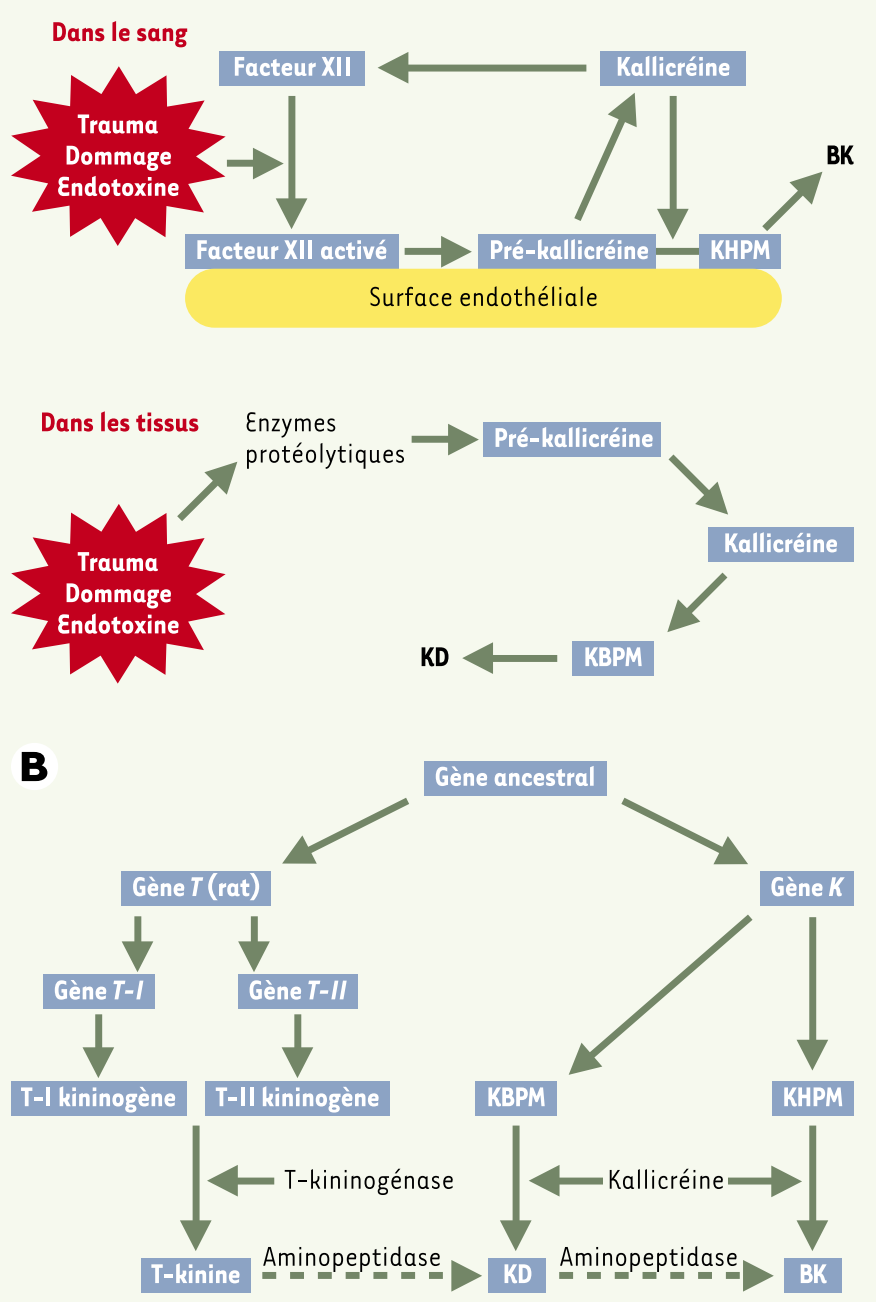

Figure 1. A. Les deux voies biochimiques principales impliquées dans la formation plasmatique et tissulaire des kinines. Dans le sang, le facteur de déclenchement est l'activation du facteur de Hageman (Facteur XII) par des surfaces endommagées. L'activation subséquente de la kallicréine libère la bradykinine (BK) du kininogène de haut poids moléculaire (KHPM). Dans les tissus, le précurseur des kinines est le kininogène de bas poids moléculaire (KBPM). Chez I'homme, la kallicréine tissulaire libère la kallidine (KD) à partir du KBPM. B. Gènes des kinines. 
métabolite final de la BK et de la DBK. L'ECA peut également transformer la DBK en BK-(1-5) dans le plasma humain [6] (Figure 2B). Enfin, la KD et la T-kinine sont transformées en BK en présence des aminopeptidases $[4,6]$.

\section{Récepteurs des kinines}

Les kinines causent leurs effets biologiques, incluant la vasodilatation, l'augmentation de la perméabilité vasculaire, la stimulation de terminaisons nerveuses sensorielles et sympathiques et la contraction de muscles lisses par

A

DÉGRADATION DES KININES

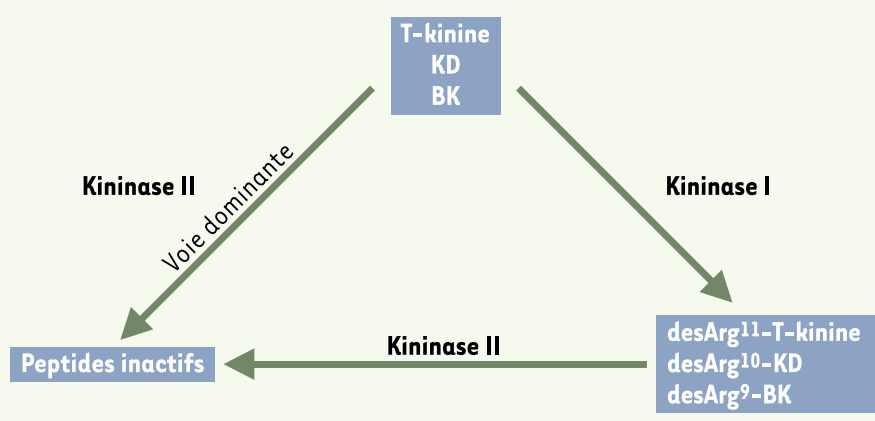

Les sites de clivage de la formation des kinines
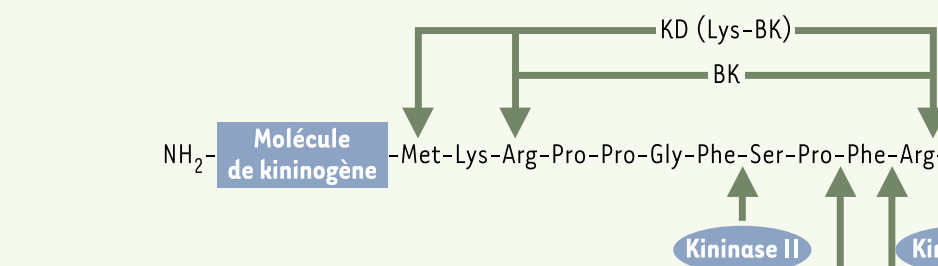

B

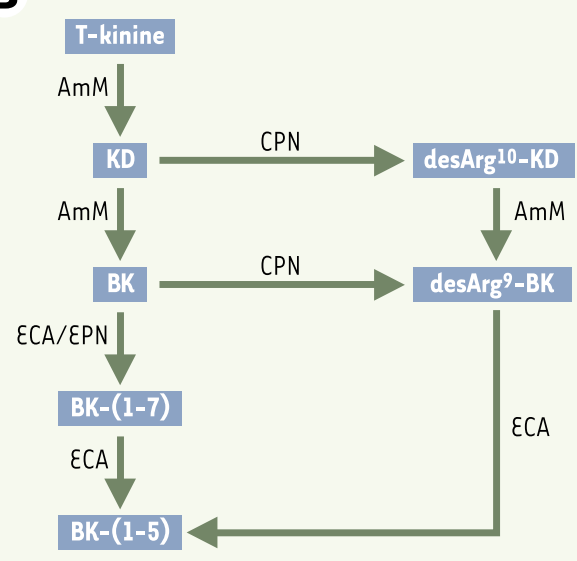

Figure 2. A. Sites de clivage au cours de la formation et de la dégradation des kinines. BK: bradykinine; KD: kallidine. B. Représentation schématique de la dégradation des kinines dans le plasma humain. AmM: aminopeptidase M; CPN : carboxypeptidase N; ECA: enzyme de conversion de l'angiotensine-I; EPN: endopeptidase neutre. l'activation de deux types de récepteurs, $B_{1}$ et $B_{2}$. Ces récepteurs ont été définis, sur la base de critères pharmacologiques, par l'usage d'agonistes et d'antagonistes peptidiques et non peptidiques [7-9]. La BK, la KD et la Tkinine sont des agonistes endogènes des récepteurs $B_{2}$, tandis que la DBK et la desArg ${ }^{10}-K D$ sont les agonistes préférentiels des récepteurs $B_{1}$.

Ces récepteurs possèdent sept domaines transmembranaires et appartiennent à la grande famille des récepteurs couplés aux protéines $G\left(G_{\alpha q} / 11\right.$ et $G_{\alpha i}$ ). La séquence des acides aminés du récepteur $B_{1}$ humain (353 acides aminés) n'est identique qu'à seulement $36 \%$ de celle du récepteur $B_{2}$ humain (364 acides aminés), tandis que l'homologie n'est que de $30 \%$ entre les récepteurs $B_{2} \quad(366$ acides aminés) et $B_{1}$ (334 acides aminés) chez la souris [4] (Tableau II).

\section{Mécanismes}

de signalisation

des récepteurs des kinines La transduction des signaux pour les récepteurs des kinines est relayée par des systèmes de seconds messagers différents selon le type cellulaire, et implique l'activation de protéines G. Parmi les mécanismes de transduction de signaux, notons l'activation directe ou indirecte de l'adénylate cyclase et de la guanylate cyclase, menant à la formation de l'adénosine monophosphate cyclique (AMPc) et de la guanosine monophosphate cyclique (GMPc), l'activation de canaux ioniques et l'activation des phospholipases $\mathrm{A}_{2}, \mathrm{C}$ et $\mathrm{D}$ (Figure 3). La phospholipase C (PLC) stimule la formation d'inositol 1,4,5-triphosphate (IP3) et du diacylglycérol (DAG), impliqués respectivement dans la libération du calcium intracellulaire et dans l'activation de la 
protéine kinase C (PKC). L'acide arachidonique peut également être produit à partir des phospholipides membranaires, via l'activité de la phospholipase $A_{2}\left(P L A_{2}\right)$ et à partir du DAG sous l'action d'une lipase, ce qui conduit à la production de prostaglandines (PG). À la suite d'une dépolarisation de la membrane, le calcium peut stimuler la formation de l'AMPc, du GMPc et du monoxyde d'azote (NO). Outre ces voies classiques, des travaux récents suggèrent que le récepteur $B_{2}$ est également lié à d'autres voies de transduction de signaux, incluant l'activation de protéines à activité tyrosine kinase cytoplasmiques [10] et l'activation de la MAP-kinase, qui succède à l'activation directe de la PKC et de Raf (une sérine/thréonine kinase) ou à la phosphorylation de la tyrosine des récepteurs du facteur de croissance épidermique (EGF) suivie de l'activation de la Ras kinase $[4,11]$. Le récepteur $B_{1}$ est principalement associé à l'activation de la PLC et à la voie des phosphoinositols, bien que l'on y retrouve aussi la $\mathrm{PLA}_{2}$ et la MAPkinase [9].

\section{Récepteur $B_{2}$ des kinines}

Le récepteur $B_{2}$ est constitutif, et responsable de la majorité des réponses pharmacologiques des kinines observées dans la phase aiguë de l'inflammation et de la douleur [2, 12]. Cette fonctionnalité aiguë des récepteurs $B_{2}$ est probablement le résultat des mécanismes rapides d'association et de dissociation du ligand au récepteur $B_{2}$, de la désensibilisation du récepteur et de son internalisation, et de la régulation négative du récepteur lors du maintien à long terme de la stimulation [2]. Le récepteur $B_{2}$ est distribué de façon ubiquitaire et est présent notamment sur les cellules endothéliales, les cellules musculaires lisses, les fibroblastes, les cellules mésangiales et épithéliales, certains neurones, les astrocytes et les polynucléaires neutrophiles [3]. Plusieurs excellents agonistes et antagonistes sélectifs du récepteur $B_{2}$, de nature peptidique ou non, ont été développés $[4,7,8]$ (Tableau III). ment la transformation de l'angiotensine I en angiotensine II, qui est un vasoconstricteur puissant, mais aussi la dégradation des kinines endogènes, connues pour leurs effets vasodilatateur, cardioprotecteur, natriurétique et diurétique [13]. Bien que le rôle des kinines endogènes dans le maintien de la pression artérielle normale soit encore mal défini, leur implication dans les effets cardioprotecteurs et antihypertenseurs des inhibiteurs de l' $€ C A$ est aujourd'hui bien accepté, tant dans des modèles expérimentaux que chez l'homme $[13,14]$. Notons que les souris dont le gène du récepteur $B_{2}$ a été invalidé ont une pression sanguine de base plus élevée que les souris de type sauvage, et développent une hypertension artérielle quand elles sont soumises à un régime riche en sel [15].

\section{Fonction du récepteur $B_{2}$ dans l'inflammation neurogénique}

Les récepteurs $B_{2}$ sont impliqués dans la plupart des signes cardinaux de l'inflammation, dont la dilatation artérielle, la veinoconstriction, l'augmentation de la perméabilité vasculaire, l'œdème et la douleur [2]. L'activation du récepteur $B_{2}$ cause une vasodilatation en raison de la stimulation de la $\mathrm{NO}$-synthase et de la $\mathrm{PLA}_{2}$, menant à la production de monoxyde d'azote (NO) et de prostacycline $\left(P \mathrm{II}_{2}\right)$ par les cellules endothéliales des vaisseaux précapillaires (vascularisation artérielle) (Figure 4) [3]. Sur les vaisseaux postcapillaires (veines et veinules), la BK cause une vasoconstriction par une action directe sur le muscle lisse (Figure 5). La vasodilatation des artérioles précapillaires et la contraction des veinules postcapillaires favorisent l'augmentation de la pression hydrostatique dans les capillaires, cause de l'extravasation plasmatique de fluides et de protéines. De plus, la BK augmente la perméabilité vasculaire par l'activation et la contraction des cellules endothéliales vasculaires. Ce mécanisme conduit à une fenestration de la paroi des microvaisseaux, principalement des veinules, favorisant encore l'extravasation plasmatique.

\section{Fonction du récepteur $B_{2}$ vasculaire}

Dans le traitement de l'hypertension et de plusieurs maladies cardiovasculaires, dont l'insuffisance cardiaque, l'infarctus du myocarde et la néphropathie diabétique, les inhibiteurs de l'enzyme de conversion de l'angiotensine (દCA) tels que l'énalapril ou le ramipril sont utilisés, avec de bons résultats. Ces inhibiteurs empêchent non seule-

\begin{tabular}{lll}
\hline & Récepteur $B_{1}$ & Récepteur $B_{2}$ \\
\hline Famille & Couplé à une protéine $G$ & Couplé à une protéine $G$ \\
\hline Nombre d'acides aminés & 353 (homme) & 364 (homme) \\
\hline Mode d'expression & Inductible & Constitutif \\
\hline Désensibilisation & Non & Oui \\
\hline Internalisation & Non & Oui \\
\hline Transduction des signaux & PLA $_{2}$, PLC, MAP-kinase & PLA, PLC, PLD, AMPc, GMPc, \\
& & canaux ioniques, PTK, MAP-kinase
\end{tabular}

$\mathrm{PLA}_{2}$ : phospholipase $\mathrm{A}_{2}$; PLC: phospholipase C; MAP-kinase: mitogen-activated protein kinase; PLD: phospholipase $D$; AMPc: adénosine monophosphate cyclique; GMPc: guanosine monophosphate cyclique; PTK: protéine tyrosine kinase.

Tableau II. Résumé des propriétés des récepteurs $B_{1}$ et $B_{2}$ des kinines. 
La BK facilite la libération de neuropeptides pro-inflammatoires tels que la substance P (SP) et le CGRP (calcitonine-gene related peptide) par les neurones sensoriels de rat [2]. La SP contribue à l'inflammation en induisant une contraction des veinules, produisant ainsi une extravasation plasmatique. La SP produit également une vasodilatation via la libération de NO par les cellules endothéliales. Le CGRP contribue à l'inflammation neurogénique en empêchant la dégradation de la SP et en produisant une dilatation des artérioles, ce qui augmente le flux sanguin. L'augmentation de la libération de neuropeptides sensoriels induite par la BK est généralement favorisée par les prostaglandines et réduite par les inhibiteurs de la cyclooxygénase [2] $(\rightarrow)$.

\section{Fonction du récepteur $B_{\mathbf{2}}$ dans la douleur inflammatoire}

L'application de BK sur la peau humaine et dans les tissus de rat cause la douleur en stimulant les récepteurs $B_{2}$. La stimulation des récepteurs $B_{2}$ présents sur les afférences sensitives primaires induit l'activation des récepteurs nociceptifs polymodaux et l'hyperalgésie par la production de DAG et l'activation de la PKC [2, 12]. De plus, la BK peut sensibiliser les nocicepteurs en stimulant la formation de prostaglandines (PG), de cytokines et de NO par les neurones sensoriels, les cellules endothéliales ou immunitaires, ou encore par les fibroblastes, outre son interaction avec les médiateurs d'origine mastocytaire comme l'histamine et la sérotonine [2, 12]. La stimulation des nerfs sympathiques peut également induire la libération de PG ou d'autres médiateurs qui sensibilisent les nocicepteurs, et contribuent ainsi à l'hyperalgésie induite par la BK. Ces résultats expérimentaux pourraient expliquer l'effet antinociceptif des antagonistes du récepteur $B_{2}$ dans des modèles d'hyperalgésie aiguë d'origine inflammatoire [16], et justifier les changements de réponses nociceptives chez les souris dont le gène du récepteur $B_{2}$ est invalidé [2].

\section{Fonction du récepteur $B_{2}$ central dans la modulation de la douleur}

Le système nerveux central contient toutes les composantes du système kallicréine-kinine. Les récepteurs $B_{2}$ ont été identifiés dans diverses régions du cerveau et de la moelle épinière de mammifères [4]. La majorité des sites de liaison identifiés par autoradiographie dans la moelle épinière sont situés dans les couches superficielles de la corne dorsale, en particulier sur les terminaisons nerveuses des fibres sensorielles $A \delta$ et $C$, et des fibres bulbospinales noradrénergiques $[2,4]$. Après administration dans les ventricules cérébraux, la BK cause un effet antinociceptif via un mécanisme noradrénergique chez le lapin, la production de NO chez la souris ou l'activation des récepteurs $B_{2}$ dans le cerveau de rat [4]. L'administration intrathécale de la BK chez le rat éveillé cause une brève excitation comportementale suivie par une plus longue période de tranquillité et par une réponse analgésique à un stimulus thermique nociceptif. La réponse nociceptive serait due à une activation des récepteurs $B_{2}$ sur les terminaisons nerveuses senso-

Figure 3. Transduction des signaux par le récepteur $B_{2}$ couplé à une protéine $G$. PKC: protéine kinase $C$; IP3: inositol 1,4,5-triphosphate; DAG : diacylglycérol; NO: monoxyde d'azote. 
rielles dans les couches superficielles de la moelle épinière, alors que la réponse antinociceptive serait tributaire d'une libération de noradrénaline par des neurones inhibiteurs descendants qui projettent à la corne dorsale, avec activation subséquente des récepteurs $\alpha_{2}$-adrénergiques [2]. La stimulation centrale des récepteurs $B_{2}$ de la $B K$ peut être un événement important dans la composante douloureuse de la migraine et dans d'autres désordres cérébrovasculaires, ainsi que dans l'inflammation neurogénique observée dans diverses formes de traumatismes cérébraux.

\section{Récepteur $B_{1}$ des kinines}

Le récepteur $B_{1}$ est généralement absent dans les tissus d'animaux sains, et exprimé chez les animaux atteints d'une infection. II est induit et surexprimé à la suite de lésions tissulaires ou de l'exposition à des endotoxines bactériennes ou à des cytokines telles que l'interleukine$1 \beta$ et le facteur de nécrose tumorale $\alpha$ (TNF- $\alpha$ ). De plus, le récepteur $B_{1}$ ne se désensibilise pas en présence de son agoniste [9]. Toutefois, chez le chien, le récepteur $B_{1}$ semble être constitutif et son activation cause une hypotension, une natriurèse et une vasodilatation rénale $[17,18]$. Des agonistes et des antagonistes peptidiques spécifiques de $B_{1}$ ont été développés [7-9] (Tableau IV). Des antagonistes non peptidiques du récepteur $B_{1}$ ne sont pas encore disponibles commercialement.

\section{Induction du récepteur $B_{1}$}

Une synergie semble exister entre le ligand du récepteur $B_{1}$ et l'interleukine- $1 \beta$ pour augmenter l'expression des récepteurs $B_{1}$ [19]. L'induction du récepteur $B_{1}$ par les cytokines est contrôlée par la MAP-kinase, le facteur de transcription nucléaire (NF-KB) et la protéine kinase $p 38$ activée par le stress [20-22]. Des études menées in vivo dans un modèle d'hyperalgésie inflammatoire suggèrent que les récepteurs $B_{1}$ peuvent également être induits par l'activation du récepteur $B_{2}$ (via la production autocrine des cytokines) et/ou sa désensibilisation par séquestration [2]. Par ailleurs, l'activation des récepteurs $B_{2}$ peut directe- ment activer le NF- $\kappa B$, qui à son tour induit l'expression des récepteurs $B_{1}$ [19]. En outre, l'invalidation du gène du récepteur $B_{2}$ chez la souris entraîne une surexpression des récepteurs $B_{1}[15,23]$. Malgré ces observations, l'existence d'un mécanisme d'autorégulation réciproque entre les récepteurs $B_{1}$ et $B_{2}$ n'est pas admise de façon consensuelle. $\varepsilon n$ effet, des études réalisées in vivo et in vitro, chez le lapin et sur des cellules en culture, montrent que ni la stimulation des récepteurs $B_{1}$ et $B_{2}$ par des ligands exogènes ou endogènes, ni leur blocage chronique par des antagonistes n'ont d'effet significatif sur leur expression mutuelle [24].

Contrairement aux récepteurs $B_{2}$, les récepteurs $B_{1}$ participent à la phase chronique de la réponse inflammatoire et de la douleur [2, 12]. Ils produisent des réponses et des signaux persistants, avec une faible désensibilisation et une internalisation limitée associée à une dissociation lente du ligand. L'activation chronique des récepteurs $B_{1}$ peut probablement être amplifiée par l'accumulation de la DBK (desArg'-BK) au site d'inflammation [2]. L'induction de la CPM (carboxypeptidase $M$ de la membrane cellulaire ou kininase I) peut également augmenter le niveau des métabolites actifs sur le récepteur $B_{1}$ dans le processus d'inflammation [2].

\begin{tabular}{|c|c|c|}
\hline & Peptidique & Non peptidique \\
\hline \multirow{3}{*}{ Agonistes } & BK & FR 190997 \\
\hline & Lys-BK (KD) & \\
\hline & $\mathrm{Phe}^{8} \Psi\left(\mathrm{CH}_{2} \mathrm{NH}\right) \mathrm{Arg}^{9}-\mathrm{BK}$ & \\
\hline \multirow[t]{5}{*}{ Antagonistes } & D-Arg $\left[\mathrm{Hyp}^{3}, \mathrm{Thi}^{5,8}, \mathrm{D}-\mathrm{Phe}^{7}\right] \mathrm{BK}$ & WIN 64338 \\
\hline & Icatibant (Hoe 140) & FR 173657 \\
\hline & Men 11270 & LF 16.0335 \\
\hline & NPC 17731 & LF 16.0687 \\
\hline & NPC 17761 & Bradyzide \\
\hline
\end{tabular}

FR 190997 8-[2,6-dichloro-3-[N-[( 2 -4- (N-méthylcarbamoyl) cinnamidoacétyl]-N méthylamino] benzyloxy]-2-méthyl-4-(2-pyridylméthoxy) quinoline

Hoe 140 D-Arg $\left[\mathrm{Hyp}^{3}, \mathrm{Thi}^{5}, \mathrm{D}\right.$-Tic, Oic $\left.{ }^{8}\right]$ BK

Men 11270 Le dérivé cyclique contraint du Hoe 140 (D-Arg-Arg-Pro-Hyp-Gly-Thi-c(Dab-D-Tic-Oic-Arg)c $(7 \gamma-10 \alpha))$

NPC 17731 D-Arg $\left.{ }^{0}\left[\mathrm{Hyp}^{3}, \mathrm{D}-\mathrm{Hyp}-\varepsilon \text { (trans-propyl) }\right)^{7}, \mathrm{Oic}^{8}\right] \mathrm{BK}$

NPC 17761 D-Arg $\left.{ }^{0}\left[\mathrm{Hyp}^{3}, \mathrm{D}-\mathrm{Hyp}-\varepsilon \text { (trans-thio-phenyl) }\right)^{7}, \mathrm{Oic}^{8}\right]$ BK

WIN 64338 (Phosphonium, [ [4-[[2[[bis(cyclohexylamino)méthylène $]$ amino]-3-(2-naphthalényl)-1-oxopropyl]amino] phényl]méthyl] tributyl, chlorure, monohydrochlorure)

FR 173657 ((ع)-3-(6-acétamido-3-pyridyl)-N-[2,4-dichloro-3-[(2-méthyl-8-quinolinyl) oxyméthyl] phényl]-N méthylaminocarboxylméthyl]-acrylamide

LF 16.0335 (1-[[3-[(2,4-diméthylquinolin-8-yl)oxyméthyl]-2,4-dichloro-phényl]-sulphonyl]-2(S)-[[4-[4(aminoiminométhyl)phényl-carbonyl]pipérazine-1 yl]carbonyl]pyrrolidine)

LF 16.0687 (1-[[2,4-dichloro-3-[[(2,4-diméthylquinolin-8-yl)oxy] méthyl] phényl]-sulphonyl]-N-[-3[[4-(aminoiminométhyl)-phényl]carbonylamino]-propyl]-2(S)-pyrrolidinecarboxamide)

Bradyzide ((2S)-1-[4-(benzhydrylthiosemicarbazido)-3-nitrobenzènesulfonyl]-pyrrolidine-2-acide carboxilique $\{2$-(2-diméthylaminométhyl)-méthylamino]éthyl\}amide)

Tableau III. Agonistes et antagonistes des récepteurs des kinines. 


\section{Rôle du récepteur $B_{1}$ dans l'œdème}

Les récepteurs $B_{1}$ et $B_{2}$ semblent être impliqués dans le développement des réponses inflammatoires locales dans des modèles d'arthrite aiguë et chronique chez le rat (œdème de la patte, extravasation des protéines menant à l'œdème de l'articulation) [2, 25]. L'induction du récepteur $B_{1}$ par les cytokines ou le lipopolysaccharide (LPS) a été étudiée dans l'œdème de la patte chez le rat [26]: cette réponse inflammatoire relayée par le récepteur $B_{1} a$ été attribuée à la libération de SP et de CGRP à partir des fibres sensorielles de type $C$, à la production de sérotonine des mastocytes et à la synthèse de PG.

\section{Rôle du récepteur $B_{1}$ dans la composante cellulaire de la réponse inflammatoire}

Les effets pro-inflammatoires du récepteur $B_{1}$ incluent également la stimulation de la migration des leucocytes sanguins. Outre les récepteurs $B_{1}$, les kallicréines et les kininogènes sont retrouvés à la surface des neutrophiles circulants et synoviaux, et représentent une façon efficace de délivrer les kinines aux sites d'inflammation [25]. L'activation du récepteur $B_{1}$ induit les trois phases du processus de recrutement des leucocytes: roulement, adhérence et migration des cellules [2].

Il semble que les agonistes $B_{1}$ agissent directement sur les neurones sensoriels pour libérer la SP et le CGRP, lesquels influencent à leur tour la chimio-attraction des neutrophiles via des récepteurs peptidiques de l'endothélium [2]. La SP et le CGRP induisent l'expression rapide des molécules d'adhérence des cellules endothéliales vasculaires $(\varepsilon-$ sélectine, $\mathrm{P}$-sélectine et la molécule d'adhérence intercellulaire-1 ou ICAM-1), lesquelles jouent un rôle fondamental dans le roulement et l'adhérence des neutrophiles circulants [2]. Alternativement, les récepteurs $B_{1}$ peuvent activer indirectement les fibres sensorielles de type $C$ par libération de $P G$, des médiateurs des mastocytes et des cytokines, particulièrement l'interleukine-l.

Le récepteur $B_{1}$ a été détecté, en immunocytochimie, sur des cellules endothéliales et périvasculaires inflammatoires issues d'échantillons de cerveau prélevés à l'autopsie chez des patients atteints de sclérose en plaques [27]. Le récepteur $B_{1}$ est induit par l'interféron- $\gamma$ sur les cellules endothéliales en culture provenant de cerveau humain. Son activation entraîne une inhibition de la libération d'interleukine- 8 par les cellules endothéliales et une augmentation de la perméabilité aux grosses molécules (albumine) d'une membrane artificielle hématoencéphalique intégrant des cellules endothéliales. De plus, une corrélation a été observée entre l'activité du récepteur $B_{1}$ et son expression sur des lymphocytes T dérivés du sang de patients atteints de sclérose en plaques [28]. Les cytokines pro-inflammatoires (interféron- $\gamma$, TNF- $\alpha$ ), dont la concentration plasmique est élevée dans la sclérose en plaques, potentialisent l'expression du récepteur $B_{1}$ à la surface des lymphocytes $T$. Dans cette même étude, l'activation du récepteur $B_{1}$ des cellules $T$ empêche leur migration à travers une membrane hématoencéphalique artificielle. Ces résultats suggèrent un rôle protecteur du récepteur $B_{1}$ dans cette maladie: en effet, le récepteur $B_{1}$ réduirait l'infiltration des cellules immunes dans le cerveau, par l'intermédiaire d'une réduction de la sécrétion d'interleukine- 8 par les cellules endothéliales et par un effet direct anti-migratoire sur les lymphocytes T. Or, ces cellules immunes jouent un rôle important dans le déclenchement du processus inflammatoire de démyéliniation observé dans la sclérose en plaques. 


\section{Rôle du récepteur $B_{1}$ dans la douleur d'origine inflammatoire}

Des études récentes en immunohistochimie ont montré une expression basale des récepteurs $B_{1}$ dans les ganglions sensoriels et dans les terminaisons nerveuses centrales et périphériques des neurones sensoriels (fibres $A \delta$ et $C$ ) chez le rat, de même que dans la corne dorsale chez l'homme [2]. Le groupe de J.B. Pesquero [29] a également montré la présence de récepteurs $B_{1}$ dans la moelle épinière de la souris, leur activation facilitant un réflexe nociceptif (une réaction à la douleur). Chez la souris dont le gène du récepteur $B_{1}$ est invalidé, il y a une réduction du phénomène de sensibilisation aux stimulus nociceptifs, mesurée au niveau de la moelle épinière, et une perte de sensibilité aux stimulus chimiques et thermiques dans les essais comportementaux de nociception [29]. De plus, J. Ferreira et al. [30] ont rapporté une diminution de l'hyperalgésie dans un modèle de douleur inflammatoire persistante chez le même type de souris.

Malgré cette expression basale des récepteurs $B_{1}$, leur rôle physiologique dans le contrôle des informations douloureuses en situation normale, chez l'animal sain, demeure encore incertain. En effet, les agonistes du récepteur $B_{1}$ des kinines n'ont pas d'effet sur la nociception chez des rats normaux ou dans des modèles aigus d'inflammation [16], et n'entraînent ni la production de seconds messagers, ni la libération de neuropeptides, ni la survenue d'événements électrophysiologiques dans des neurones sensoriels soumis en conditions témoins ou inflammatoires aiguës $[2,12]$. Les antagonistes pharmacologiques du récepteur $B_{1}$ induisent une analgésie seulement dans des modèles animaux d'hyperalgésie mécanique et thermique persistante, d'origine inflammatoire, ou dans la douleur persistante viscérale. Ces résultats peuvent être expliqués par l'induction des récepteurs $B_{1}$ sur des cellules autres que les neurones sensoriels (macrophages, fibroblastes ou les cellules endothéliales), où ils peuvent être responsables de la libération de médiateurs ( $P G$, cytokines et NO) sensibilisant ou activant les nocicepteurs [2, $12]$.

Dans un modèle d'hypersensibilité neuropathique causée par une lésion des nerfs périphériques chez le rat, les antagonistes des récepteurs $B_{1}$ ont un effet analgésique mesurable seulement 14 jours après la lésion, alors que les antagonistes du récepteur $B_{2}$ induisent une analgésie mesurable 48 h et 14 jours après [31]. Dans l'étude précédente, ainsi que dans deux études semblables, une augmentation de l'expression (ARNm) des récepteurs $B_{1}$ et $B_{2}$ des kinines a été mise en évidence dans les ganglions sensoriels de la racine dorsale deux jours après ligature du nerf sciatique chez le rat, augmentation qui pourrait contribuer à l'hyperalgésie consécutive à une douleur inflammatoire [2]. Ainsi, une activation directe du récepteur $B_{1}$ des neurones sensoriels par les kinines endogènes est possible; cet effet peut être sensibilisé par l'action des PG ou de médiateurs libérés à partir d'autres cellules par l'activation de l'un ou l'autre type de récepteurs de la BK.

Les données expérimentales actuelles suggèrent donc que les récepteurs $B_{1}$ sont principalement impliqués dans la douleur persistante inflammatoire, leur rôle potentiel dans le contrôle de la douleur aiguë chez l'homme restant à démontrer.

\section{Rôle du récepteur $B_{1}$ dans le diabète}

Des données expérimentales suggèrent que le diabète est une autre condition pathologique pouvant entraîner l'induction des récepteurs $B_{1}$. D'après les connaissances actuelles, le diabète sucré de type 1 , ou insulinodépendant, est dû à une réponse auto-immune impliquant la surproduction de cytokines, dont l'interleukine- $1 \beta$ et le TNF$\alpha$, qui mène à la destruction des cellules $\beta$ des îlots pancréatiques [32]. L'hyperglycémie et le stress oxydatif en résultant peuvent aussi activer NF-KB [33], que l'on sait capable d'induire le récepteur $B_{1}$ [9]. Ainsi, la surproduction de cytokines et l'hyperglycémie pourraient déclencher, au cours du diabète, l'expression du récepteur $B_{1}$ par l'intermédiaire du NF-KB.

Le modèle de diabète induit par la streptozotocine (STZ) est le plus couramment utilisé pour étudier les complications cardiovasculaires et neuropathiques du diabète. La STZ est un antibiotique extrait de Streptomyces acromogenes, sélectivement toxique pour les cellules $\beta$ des îlots pancréatiques où il cause une inflammation impliquant la libération de cytokines [34]. Des données pharmacologiques suggèrent que le récepteur $B_{1}$ intervient dans la pathogénie du diabète induit par la STZ chez les souris, et que l'insulinite est une réaction inflammatoire lente

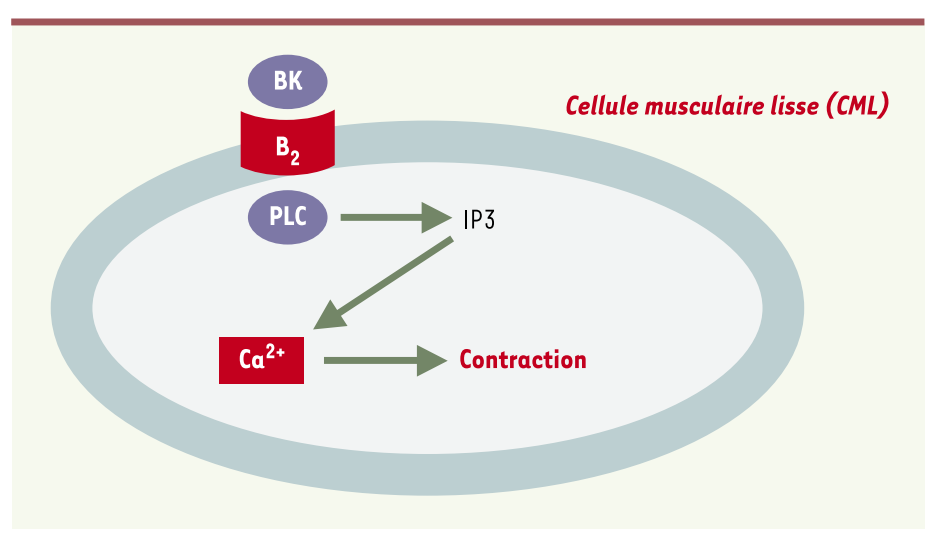

Figure 5. Mécanisme d'action de la BK au niveau des vaisseaux post-capillaires. Le récepteur $B_{2}$ serait présent à la surface des cellules musculaires lisses de ces vaisseaux. Son activation entraîne la stimulation de la PLC, la production de I'IP3 et la libération de $\mathrm{Ca}^{2+}$ intracellulaire conduisant à la contraction des CML et des vaisseaux post-capillaires. 
6. Kuoppala A, Lindstedt KA, Saarinen J, Kovanen PT, Kokkonen J0. Inactivation of bradykinin by angiotensin-converting enzyme and by carboxypeptidase $\mathrm{N}$ in human plasma. Am J Physiol Heart Circ Physiol 2000; 278: H1069-74.

7. Regoli D, Nsa Allogho S, Rizzi A, Gobeil FJ. Bradykinin receptors and their antagonists. Eur J Pharmacol 1998; 348: 1-10.

8. Regoli D, Rizzi A, Perron SI, Gobeil F. Classification of kinin receptors. Biol Chem 2001 ; 382: 31-5.

9. Marceau F, Hess JF, Bachvarov DR. The Bl receptors for kinins. Pharmacol Rev 1998; 50: 357-86.

10. Velarde V, Ullian ME, Morinelli TA, Mayfield RK, Jaffa AA. Mechanisms of MAPK activation by bradykinin in vascular smooth muscle cells. Am J Physiol 1999; 277: C253-61.

11. Adomeit A, Graness A, Gross S, Seedorf K, Wetzker $R$, Liebmann C. Bradykinin $B_{2}$ receptor-mediated mitogen-activated protein kinase activation in COS-7 cells requires dual signaling via both protein kinase $C$ pathway and epidermal growth factor receptor transactivation. Mol Cell Biol 1999; 19: 5289-97.

12. Dray A. Kinins and their receptors in hyperalgesia. Can J Physiol Pharmacol 1997; 75: 704-12.

13. Linz W, Wiemer G, Gohlke P, Unger T, Scholkens BA. Contribution of kinins to the cardiovascular actions of angiotensin-converting enzyme inhibitors. Pharmacol Rev 1995; 47: 25-49.

14. Rhaleb NE, Yang XP, Nanba $M$, Shesely $\varepsilon G$, Carretero $O A$. Effect of chronic blockade of the kallikrein-kinin system on the development of hypertension in rats. Hypertension 2001; 37: 121-8.
15. Duka I, Kintsurashvili $\varepsilon$, Gavras I, Johns C, Bresnahan M, Gavras H. Vasoactive potential of the $B_{1}$ bradykinin receptor in normotension and hypertension. Circ Res 2001; 88: 275-81.

16. Calixto JB, Cabrini DA, Ferreira J, Campos MM. Kinins in pain and inflammation. Pain 2000; 87: 1-5.

17. Lortie M, Regoli D, Rhaleb $N \varepsilon$, Plante $G \varepsilon$. The role of $B_{1}$ - and $B_{2}$-kinin receptors in the renal tubular and hemodynamic response to bradykinin. Am J Physiol 1992; 262: R72-6.

18. Nakhostine $N$, Ribuot $C$, Lamontagne D, Nadeau R, Couture R. Mediation by $B_{1}$ and $B_{2}$ receptors of vasodepressor responses to intravenously administered kinins in anaesthetized dogs. BrJ Pharmacol 1993; 110: 71-6.

19. Phagoo SB, Poole S, LeebLundberg LM. Autoregulation of bradykinin receptors: agonists in the presence of interleukin-lbeta shift the repertoire of receptor subtypes from $B_{2}$ to $B_{1}$ in human lung fibroblasts. Mol Pharmacol 1999; 56 : 325-33.

20. Larrivée JF, Bachvarov DR, Houle F, Landry J, Huot J, Marceau F. Role of the mitogen-activated protein kinases in the expression of the kinin $B_{1}$ receptors induced by tissue injury. J Immunol 1998; 160: 1419-26.

21. Campos MM, Souza GEP, Calixto JB. In vivo $B_{1}$ kinin receptor upregulation. Evidence for involvement of protein kinases and nuclear factor $-\kappa B$ pathways. BrJ Pharmacol 1999; 127 : 1851-9.

22. Ganju P, Davis A, Patel $S$, Nunez $X$, Fox A. p38 stressactivated protein kinase inhibitor reverses bradykinin $B_{1}$ receptor-mediated component of inflammatory hyperalgesia. Eur Pharmacol 2001; 421: 191-9.
23. Marin-Castano ME, Schanstra JP, Neau $E$, et al. Induction of functional bradykinin $B_{1}$ receptors in normotensive rats and mice under chronic angiotensinconverting enzyme inhibitor treatment. Circulation 2002; 105: 627-32.

24. Sabourin T, Guay K, Houle $S$, et al. Absence of ligandinduced regulation of kinin receptor expression in the rabbit. BrJ Pharmacol 2001; 133: 1154-62.

25. Bhoola K, Ramsaroop R, Plendl J, Cassim B, Dlamini Z, Naicker S. Kallikrein and kinin receptor expression in inflammation and cancer. Biol Chem 2001; 382: 77-89.

26. Campos MM, Souza GE, Calixto JB. Modulation of kinin $B_{1}$ but not $B_{2}$ receptors-mediated rat paw edema by IL-lbeta and TNFalpha. Peptides 1998; 19: 1269-76.

27. Prat A, Biernacki K, Pouly S, Nalbantoglu J, Couture R, Antel JP. Kinin $B_{1}$ receptor expression and function on human brain endothelial cells. J Neuropathol Exp Neurol 2000; 59: 896-906.

28. Prat A, Weinrib L, Becher B, et al. Bradykinin $B_{1}$ receptor expression and function on T lymphocytes in active multiple sclerosis. Neurology 1999; 53: 2087-92.

29. Pesquero JB, Araujo RC, Heppenstall PA, et al. Hypoalgesia and altered inflammatory responses in mice lacking kinin $B$ receptors. Proc Natl Acad Sci USA 2000; 97 : 8140-5.

30. Ferreira J, Campos MM, Pesquero JB, Araujo RC, Bader M, Calixto JB.

Evidence for the participation of kinins in Freund's adjuvant-induced inflammatory and nociceptive responses in kinin $B_{1}$ and $B_{2}$ receptor knockout mice. Neuropharmacology 2001; 41: 1006-12.
31. Levy D, Zochodne DW Increased mRNA expression of the $B_{1}$ and $B_{2}$ bradykinin receptors and antinociceptive effects of their antagonists in an animal model of neuropathic pain. Pain 2000; 86: 265-71.

32. Rabinovitch $A$. An update on cytokines in the pathogenesis of insulindependent diabetes mellitus. Diabetes Metab Rev 1998; 14: 129-51.

33. Yerneni KK, Bai W, Khan BV, Medford RM, Natarajan R. Hyperglycemia-induced activation of nuclear transcription factor kappa $B$ in vascular smooth muscle cells. Diabetes 1999; 48: 855-64.

34. Zuccollo A, Navarro $M$, Catanzaro 0 . Effects of $B_{1}$ and $B_{2}$ kinin receptor antagonists in diabetic mice. Can J Physiol Pharmacol 1996; 74: 586-9.

35. Mage $M$, Pécher $C$, Neau, $\varepsilon$, et al. Induction of $B_{1}$ receptors in streptozotocin diabetic rats: possible involvement in the control of hyperglycemia-induced glomerular MAP-kinase activation. Can J Physiol Pharmacol 2002; 80: 328-33.

36. Cloutier F, Couture R. Pharmacological characterization of the cardiovascular responses elicited by kinin $B_{1}$ and $B_{2}$ receptor agonists in the spinal cord of streptozotocin-diabetic rats. Br J Pharmacol 2000; 130: 375-85. 